\title{
Neuropsychological Assessment in Autism Spectrum Disorder
}

\author{
Megan L. Braconnier ${ }^{1,2}$ Paige M. Siper ${ }^{1}[$
}

Accepted: 14 July 2021 / Published online: 2 July 2021

(c) The Author(s), under exclusive licence to Springer Science+Business Media, LLC, part of Springer Nature 2021

\begin{abstract}
Purpose of Review Neuropsychological assessment involves the comprehensive evaluation of intellectual, attentional, executive, social-cognitive, language, and motor functioning. Such assessments are used to characterize areas of strength and weakness, inform differential diagnosis, guide treatment planning, and evaluate change over time. Individuals with autism spectrum disorder (ASD) present with varied clinical presentations, which can make the design of testing batteries and subsequent interpretation of results challenging. Here we provide an overview of neuropsychological domains as they relate to the evaluation of individuals with ASD.

Recent Findings Individuals with ASD demonstrate unique patterns of neuropsychological functioning across various domains. Recent findings related to intellectual, adaptive, executive, attentional, social, language, motor, and autism-specific functioning are reviewed.

Summary Clarifying the relationship between ASD symptoms and neuropsychological functioning is critical for differential diagnosis and for optimal treatment planning. Tools and methods for developing appropriate neuropsychological testing protocols for individuals with ASD are discussed.
\end{abstract}

Keywords Autism spectrum disorder · Assessment $\cdot$ Neuropsychology $\cdot$ Neuropsychological evaluation

\begin{tabular}{|c|c|}
\hline \multicolumn{2}{|c|}{ Abbreviations } \\
\hline ADI-R & Autism Diagnostic Interview-Revised \\
\hline ADOS-2 & $\begin{array}{l}\text { Autism Diagnostic Observation Schedule, } \\
\text { 2nd Edition }\end{array}$ \\
\hline BASC-3 & $\begin{array}{l}\text { Behavior Assessment System for Children, } \\
\text { 3rd Edition }\end{array}$ \\
\hline Bayley-III & $\begin{array}{l}\text { Bayley Scales of Infant and Toddler Devel- } \\
\text { opment, 3rd Edition }\end{array}$ \\
\hline BOSA & Brief Observation of Symptoms of Autism \\
\hline BRIEF & $\begin{array}{l}\text { Behavior Rating Inventory of Executive } \\
\text { Function }\end{array}$ \\
\hline CARS-2 & Childhood Autism Rating Scale, 2nd Edition \\
\hline CELF-5 & $\begin{array}{l}\text { Clinical Evaluation of Language Fundamen- } \\
\text { tals, 5th Edition }\end{array}$ \\
\hline Conners-3 & Conners 3rd Edition \\
\hline
\end{tabular}

This article is part of the Topical collection on Autism Spectrum Disorders

Paige M. Siper

paige.siper@mssm.edu

1 Seaver Autism Center for Research and Treatment, Icahn School of Medicine At Mount Sinai, New York, NY, USA

2 St. John's University, New York, NY, USA
CPT-3 Conners Continuous Performance Test, 3rd Edition

DAS-II Differential Ability Scales, 2nd Edition

D-KEFS Delis-Kaplan Executive Function System

Leiter-3 Leiter International Performance Scales-3

MSEL Mullen Scales of Early Learning

NEPSY-II NEPSY, 2nd Edition: A Developmental

Neuropsychological Assessment

PEP-3 Psychoeducational Profile, 3rd Edition

RBS-R Repetitive Behavior Scale - Revised

RPM Raven's Progressive Matrices

SAND Sensory Assessment for Neurodevelopmental Disorders

SB-5 Stanford-Binet Intelligence Scales, 5th Edition

SEQ Sensory Experiences Questionnaire

SP-2 Sensory Profile 2

SRS-2 Social Responsiveness Scale, 2nd Edition

UNIT-2 Universal Nonverbal Intelligence Test, 2nd Edition

Vineland-3 Vineland Adaptive Behavior Scales, 3rd Edition

WAIS-IV Wechsler Adult Intelligence Scale, 4th Edition 


$\begin{array}{ll}\text { WASI-II } & \begin{array}{l}\text { Wechsler Abbreviated Scale of Intelligence, } \\ \text { 2nd Edition } \\ \text { Wechsler Intelligence Scale for Children, 5th }\end{array} \\ \text { WISC-V } & \begin{array}{l}\text { Edition } \\ \text { Wechsler Preschool and Primary Scale of } \\ \text { Intelligence, 4th Edition }\end{array}\end{array}$

\section{Introduction}

Autism spectrum disorder (ASD) is a neurodevelopmental disorder characterized by deficits in social communication and interaction, as well as restricted, repetitive behaviors [1]. In addition to core symptoms of ASD, this heterogenous population presents with a wide range of abilities. While many individuals on the autism spectrum demonstrate average to above average intellectual functioning, approximately $30 \%$ also meet criteria for intellectual disability (ID) [2]. Furthermore, an estimated $60-70 \%$ of children and $69-79 \%$ of adults with ASD meet criteria for at least one comorbid psychiatric condition, such as attention-deficit/hyperactivity disorder (ADHD), anxiety, or other mood disorders [3-5]. The complexity of presentation that results from a broad spectrum of functioning, and varied behavioral and emotional presentations, can make the neuropsychological assessment process for individuals with ASD challenging and at times overlooked. Clinically, many ASD-focused evaluations include measures such as the Autism Diagnostic Observation Schedule, 2nd Edition (ADOS-2) [6] and the Autism Diagnostic Interview-Revised (ADI-R) [7], along with a measure of IQ and adaptive behavior as a standard evaluation protocol; however, fewer individuals on the autism spectrum are administered more traditional neuropsychological assessments leaving domains such as memory, attention, and other executive functions less frequently characterized. In order to fill this assessment gap, it is critical for clinicians to understand the utility and appropriate application of various neuropsychological assessments for use in individuals with ASD at varying levels of ability and to gain competence in proper interpretation based on research using neuropsychological assessments in this population.

This paper provides an overview of recent literature and concludes with recommendations for the development of appropriate neuropsychological testing protocols.

\section{Intellectual and Adaptive Functioning}

Impairment in cognitive functioning is widely recognized as a common feature of ASD, as well as a predictor of longterm outcomes [8]. The prevalence of comorbid ID has been shown to vary depending on demographic variables (e.g., location, race/ethnicity) and methodology used to assess IQ
[2], underscoring the need for clinicians to understand the tests best suited to assess IQ in autistic individuals at varying levels of ability.

The Wechsler Intelligence Scales [9-12] are among the most frequently used measures for assessing intelligence [13]. Depending on the individual's age, the Wechsler subtests examine verbal comprehension, visual-spatial reasoning, fluid reasoning, working memory, and processing speed. A recent systematic review examining multiple versions of the Wechsler Intelligence Scale for Children (WISC) in youth with ASD identified common patterns of cognitive functioning, including the "islets of ability" profile (i.e., low verbal comprehension scores and high visual-spatial and working memory scores [14]) in those with Full-Scale IQ (FSIQ) scores $\leq 85$ and the "right-descending" profile (i.e., high verbal comprehension and visual-spatial scores and low processing speed scores) in those with FSIQ scores $\geq 86$ [15•]. Similarly, adults with ASD have been shown to demonstrate deficits in processing speed and verbal reasoning subtests of the WAIS-IV, as well as inconsistent skills on perceptual reasoning tasks [16]. In addition to the Wechsler Scales, other measures of intelligence frequently used for individuals with ASD include the Stanford-Binet Intelligence Scales, Fifth Edition (SB-5) [17] and the Differential Ability Scales-II (DAS-II) [18]. The SB-5 demonstrated strong convergent validity with corresponding indices of the WISC-IV in youth with ASD, though FSIQ scores were found to be higher and scores on verbal subtests were found to be lower on the SB-5 compared to the WISC-IV [19]. While the DAS-II is often used to characterize intelligence in children with ASD, recent work by Clements et al. suggests that the verbal and nonverbal reasoning composite scores are more valid than the spatial ability composite score, which underestimates ability in this population [20].

The above-mentioned assessments are not appropriate for children below 2 years of age, and many of the tests require verbal responses, making the measures described above illsuited for individuals with few to no words. Soorya et al. provide a framework for the neuropsychological evaluation of individuals with profound intellectual and multiple disabilities, which emphasizes the need for (1) a multi-informant and multi-assessment testing battery, (2) the assessment of comorbid symptoms prior to developing an appropriate testing battery, (3) the use of unstandardized approaches to evaluate intellectual functioning, and (4) the understanding that individuals with profound intellectual and multiple disabilities are not "untestable" due to lack of standardized measures for this population [21]. Developmental assessments, such as the Mullen Scales of Early Learning (MSEL) [22], the Bayley Scales of Infant and Toddler Development, 3rd Edition (Bayley-III) [23], and the Psychoeducational Profile, 3rd Edition (PEP-3) [24], may be more appropriate for younger children with ASD and individuals with 
lower language abilities for whom valid scores cannot be obtained using traditional cognitive assessments. Nonverbal assessments, such as the Leiter International Performance Scales-3 (Leiter-3) [25], have also been recommended [26]; however, many tasks on the Leiter-3 require matching and pointing skills. As scores on cognitive versus developmental assessments have been shown to vary significantly as a function of cognitive ability [27], interchanging or comparing resulting IQ scores is not recommended. Using developmental quotients (DQ; the ratio of mental age to chronological age) in place of IQ allows for comparison between different assessments and accounts for the inability of individuals with cognitive and developmental delays to obtain standard scores using available norms [28].

Adaptive functioning is defined as one's ability to be self-sufficient in executing real-life skills, such as effectively communicating and interacting with others, managing one's own health and hygiene, and completing household tasks and chores, given their cognitive level [29, 30]. While traditional neuropsychological evaluations do not routinely administer measures of adaptive behavior, low adaptive functioning is a diagnostic criterion of ID [1] and is a common deficit in individuals with ASD [29], including those without ID [30]. Measuring adaptive functioning is therefore an important component of the evaluation process for individuals on the autism spectrum of all levels of ability. The Vineland Adaptive Behavior Scales, Third Edition (Vineland-3) [31], is a leading instrument for assessing adaptive behavior in the diagnosis of intellectual and developmental disabilities. As this measure relies on informant-report as opposed to self-report, it is appropriate for the assessment of those with lower language and cognitive abilities. Importantly, the most recent version of the Vineland-3 generally produces lower scores than the previous version of the measure, the Vineland-II [32], limiting interpretability of changes in scores between different editions. The Adaptive Behavior Assessment System, 3rd Edition (ABAS-3) [33], is a questionnaire also commonly used to assess adaptive behavior in individuals with ASD. This measure is available in parent/caregiverand teacher-report versions, as well as a self-report option for adults. The ABAS-3 has recently been found to yield higher overall scores compared to the Vineland-3 in youth with ASD without ID [34].

\section{Executive Function}

Executive functions are mental processes that direct an individual's thoughts, actions, and emotions, particularly during active problem solving. These functions include, but are not limited to, skills such as discerning appropriate goals for a particular task, planning and organizing an approach to problem solving, inhibiting distractions, initiating a task, using abstract reasoning, and demonstrating flexibility in trying a new approach [35]. While the characterization of executive function in ASD is important to determine appropriate interventions and accommodations, the literature on executive function strengths and weaknesses in this population remains mixed. A recent meta-analysis by Demetriou et al. examined executive function abilities across various domains as well as the clinical utility of measures used to assess executive function in autistic individuals [36•]. Across 235 studies comprising more than 14,000 participants, a moderate overall effect for reduced executive function was found for individuals with ASD across all key domains (Concept Formation/Set Shifting, Mental Flexibility/Set Switching, Fluency, Planning, Response Inhibition, and Working Memory) and ages, suggesting broad and stable executive function deficits [36•].

Various standardized assessment batteries have been used to assess executive function profiles in individuals on the autism spectrum, including the Delis-Kaplan Executive Functioning System (D-KEFS) [37] and the NEPSY-II [38]. The D-KEFS is a comprehensive assessment comprised of tasks related to conceptual flexibility, monitoring, and inhibition [39]. Significant executive function deficits have been reported in individuals with ASD, particularly in the areas of cognitive flexibility and response inhibition [40]. Similarly, on the NEPSY-II, a comprehensive neuropsychological battery that assesses six domains of cognitive functioning, including executive function, found that individuals with autism without ID displayed particular difficulties on tasks such as sorting, cognitive flexibility, and auditory attention [38]. Recent work by Lai et al. compared executive function profiles across studies utilizing a variety of behavioral assessments, including full test batteries such as the D-KEFS and NEPSY-II, as well as measures of individual aspects of executive function (e.g., Go-No-Go tasks, Stroop Color Word Test, Wisconsin Card Sorting Test), and found significant executive function deficits in individuals with ASD consistent with prior literature in the domains of flexibility, planning, generativity, and working memory [35]. However, further work is needed to establish the reliability of such measures in ASD populations [36•], as well as appropriate modifications for those with ASD and ID or limited expressive language.

Notably, Demetriou et al. found that very few measures of executive function achieved clinical sensitivity in ASD samples (determined by the overlap percentage statistic [OL\%] representing the degree that the performance of the ASD group overlaps with the control group, with $\mathrm{OL}<15 \%$ indicating clinical sensitivity) [36•]. Of the measures that met specifications for clinical sensitivity, most were informant-report questionnaires based on the Behavior Rating Inventory of Executive Function (BRIEF-2) [41], a questionnaire available in parent-, teacher-, and self-report 
versions assessing inhibitory self-control, flexibility, and emergent metacognition across contexts. While informantreport measures offer valuable clinical information, they pose challenges for accurate assessment in the absence of direct observation. As executive functioning is an umbrella term that encompasses a wide range of skills, ratings across instruments may be impacted by differences in the specific constructs measured. Further, many domains of executive dysfunction overlap with common ASD symptomatology (e.g., cognitive inflexibility, difficulty with inhibition), leading to diagnostic overshadowing such as the tendency to attribute co-occurring behaviors to the ASD diagnosis. The challenge teasing apart ASD symptoms from problems related to weak executive functioning further limits the appropriateness of informant-report, particularly in the assessment of individuals with lower cognitive or language abilities for whom many questions on the BRIEF are not applicable. For this reason, it is important to consider a combination of parent- and teacher-report of executive function together with direct observation using clinical interviews and standardized neuropsychological assessments.

\section{Attention}

Many domains of neuropsychological functioning, including intelligence and executive function, are impacted by attentional capacity. While previous versions of the Diagnostic and Statistical Manual of Mental Disorders (DSM) did not allow for a dual diagnosis of autism and ADHD, attentional difficulties are now recognized as one of the most common comorbidities, with ADHD prevalence in people with ASD falling in the $40-70 \%$ range [42]. Those with more severe forms of ASD have been found to present with more significant deficits in attention and behavior regulation [43]. The co-occurrence of ASD and attention problems is also associated with decreased adaptive skills [44], making the assessment and treatment of ADHD symptoms in this population essential for improving long-term outcomes.

In order to better understand the unique neuropsychological assessment needs of individuals on the autism spectrum with and without attention deficits, a recent study by $\mathrm{Ng}$ et al. administered standardized assessments of intellectual, attentional, social, and executive function to participants with ASD, ADHD, and comorbid ASD + ADHD and found differing symptom profiles depending on domain and informant [45•]. While parent-report on the Conners 3 [46], a questionnaire that measures domains such as attention and hyperactivity, differentiated individuals with ASD from those with $\mathrm{ADHD}$ or ASD + ADHD, an objective attention task (i.e., the Conners Continuous Performance Test, 2nd Edition [47]) did not [45•]. Conversely, objective assessment of social functioning and affect (i.e., NEPSY-II Affect Recognition and
Theory of Mind tasks [38]) better differentiated both ASD groups from the ADHD alone group, with the ASD groups showing greater deficits in social perception, while parentreport of social skills on the Social Responsiveness Scale, 2nd Edition (SRS-2 [48]), did not [45•]. Interestingly, no significant group differences were identified related to executive function as measured by the D-KEFS Tower Test or the Children's Category Test [45•]. This study emphasizes the benefits of integrating information from multiple sources to differentially assess ASD and attention problems, as well as the co-occurrence of the two. Inconsistencies across measures also indicate diagnoses should not be made based on results from any single instrument.

As attention and behavioral problems are positively related to ASD severity [43], it is important to assess the extent to which inattention and hyperactivity may interfere with cognitive testing of individuals with ASD. Inattention in ASD can impact performance on IQ tests, particularly on measures of processing speed and working memory [13], making the SB-5 a preferred measure of IQ in certain circumstances. In addition to caregiver- and teacher-report on measures such as the Conners 3 and the Behavior Assessment System for Children, 3rd Edition (BASC-3 [49]), which have both been shown to differentiate ASD from ADHD [45•,50], subtests of the NEPSY-II that target attention and simple behavioral tasks, such as the Conners Continuous Performance Test, 3rd Edition (CPT 3 [51]), may provide clinical utility for characterizing attention problems in this population. Future studies should also investigate sex differences, as there is evidence from parent-report on the Conners 3 indicating males with ASD exhibit higher levels of inattention and hyperactivity-impulsivity compared to females [52].

\section{Social Cognition and Affective Functioning}

Social cognition focuses on how individuals process and understand interactions with others. As social communication is a core deficit of ASD [1], assessing the extent to which an individual's social cognition impacts neuropsychological functioning is important. The main areas of social cognition typically assessed during a neuropsychological evaluation include theory of mind, emotion perception, social perception, social knowledge, and attributional style [13].

Recent work on the neuropsychological assessment of social cognition in autistic individuals has focused on the relationship between social cognition and both social and executive functioning. Bishop-Fitzpatrick et al. examined social cognition, measured by various theory of mind tasks, as a correlate of social functioning, measured by social domains of the Vineland-3 and the Child Behavior Checklist (CBCL [53]), and found better social cognitive skills 
to be associated with greater social functioning [54]. This was particularly true for participants who performed well on second-order false belief tasks, which assess the extent to which one can recognize that others can hold false beliefs about someone else's beliefs [54]. Difficulty with metacognitive aspects of executive function, measured by the NEPSYII and the BRIEF, has also been shown to be associated with deficits in social functioning in those with ASD, including decreased engagement with peers, increased playground isolation, and increased social problems $[55,56]$. In order to optimally target social impairment in individuals with ASD, it is important to consider challenges in social cognition and social behavior as they relate to executive function skills.

The NEPSY-II also includes a Social Perception domain, which has been examined in individuals with ASD [57]. This domain includes two subtests targeting affect recognition and theory of mind skills, with tasks requiring both verbal and non-verbal (i.e., matching) responses that can be individually administered depending on functioning level [38]. As with other areas of neuropsychological assessment, it is important to consider informant-report of social functioning, such as the Socialization domain of the Vineland-3. In addition, assessment of social cognition and behavior in autistic individuals generally includes autism-specific measures, such as the SRS-2, a questionnaire examining social behavior; however, the SRS-2 may not be appropriate for use with more severely affected individuals on the autism spectrum, as co-occurring behavior problems, expressive language, and cognitive abilities can significantly impact total scores [58]. Additional autism-specific diagnostic assessments, which will be discussed in a later section, can also be used to examine social skills.

\section{Language}

Language abilities are an important consideration related to the diagnostic and neuropsychological evaluation of individuals with ASD. At least $30 \%$ of children with ASD do not acquire spoken language [59] and minimally verbal individuals with co-occurring ID are more likely to exhibit poor outcomes across cognitive, adaptive, and motor domains [26]. DiStefano et al. posits that measuring general verbal and nonverbal abilities (as opposed to more specific cognitive domains) is the best method for evaluating intelligence in individuals with language and cognitive delays [26]. As quantifying verbal ability is critical to the development of appropriate treatment recommendations, measures of expressive (e.g., Expressive Vocabulary Test, 3rd Edition [60]) and receptive (e.g., Peabody Picture Vocabulary Test, 5th Edition [61]) vocabulary are important aspects of neuropsychological batteries and are worth attempting even in children with minimal language. The Clinical Evaluation of Language Fundamentals, Fifth Edition (CELF-5 [62]), is a flexible battery of tests to assess language ability and has demonstrated acceptable internal consistency for youth with ASD [63]. The CELF-5 includes certain subtests that require only pointing without verbal responses, as well as subtests that require verbal or written responses. Expressive and receptive language subtests from the Vineland-3 and the Mullen are also useful in establishing age equivalents.

\section{Motor Functioning}

Motor planning, which involves the ability to conceive, plan, and execute a purposeful motor action, is an integral developmental skill and a widely recognized deficit in individuals with ASD [13]. Individuals with ASD present with a range of motor challenges ranging from hypotonia to poor coordination. Motor slowing and other fine motor difficulties can also impact performance on timed tasks that include a motor component. It is therefore necessary to consider the potential impact of motor planning and motor slowing on an individual's performance on standardized assessments.

A recent study examining the relationship between motor skills and intelligence in children with ASD demonstrated a positive association between motor functioning and performance on neuropsychological measures [64]. In an examination of motor abilities using the Movement Assessment Battery for Children (MABC-2 [65]) and the Beery-Buktenica Developmental Test of Visual-Motor Integration (Beery VMI-6 [66]) in youth with ASD, 20.8\% of variance in performance IQ on the WISC-V was explained by variance in motor skills, with significant associations between co-occurring ID and reduced scores on the MABC-2 across domains of manual dexterity, aiming and catching, and balance [64]. Given this association, results from tasks dependent on motor planning (e.g., Block Design and Coding on WISCV) must be interpreted with caution when administered in the presence of potential motor deficits. Children with ASD have also displayed difficulty with control and regulation of handwriting movements [67], which may impact results from standardized tests requiring written responses. In conjunction with a thorough evaluation of motor skills, the identification of motor deficits can aid in decision making around an appropriate assessment battery, such as choosing assessments with fewer motor demands.

In terms of measuring motor skills, tests such as the Beery VMI-6 and Purdue Pegboard are commonly used to measure visuomotor integration [68] and goal-directed movements, including finger dexterity, hand-eye coordination, and bimanual coordination [69] in individuals on the autism spectrum. Both measures are appropriate for use with individuals with comorbid ID who are able to comprehend task instructions. Informant-report on the Vineland-3 can 
also be used to gather information about motor functioning; however, the motor domains of the Vineland-3 are currently only normed through age 9 . The Mullen provides both an assessment of early motor skills and measures of verbal and visual reception that require less motor planning than traditional intelligence tests, making the assessment of motor skills using these measures useful for individuals with cognitive and motor delays. Targeting motor skills is critical to promoting optimal development in other areas of daily functioning.

\section{Diagnostic Assessment}

In addition to the domains outlined above, the neuropsychological assessment of individuals with or suspected to have ASD should also include autism-specific diagnostic assessments to confirm diagnosis, clarify level (severity) of ASD, and to determine personal strengths and weaknesses. ASD diagnostic testing often includes standardized semi-structured observational assessments, such as the
ADOS-2 or the Childhood Autism Rating Scale, Second Edition (CARS-2 [70]), and the ADI-R, a standardized semi-structured interview administered to caregivers to assess developmental history and current autism symptoms. Further, the Social Communication Questionnaire (SCQ [71]) is a brief parent-report measure based on the ADI-R that evaluates communication skills and social functioning. Autism-specific questionnaires, such as the SRS-2, screen for ASD symptoms and provide additional data related to social functioning.

Due to limitations to in-person assessment during the COVID-19 pandemic, the Brief Observation of Symptoms of Autism (BOSA [72]), an adaptation of the ADOS-2, was recently developed to assess ASD either remotely via telehealth or in-person with the parent leading the interaction with the child. Additionally, the CARS-2 was recently adapted to include only items scored based on a clinical observation in an effort to expedite the clinician's ability to diagnose ASD [73]. The CARS-2 ${ }^{\text {obs }}$ demonstrated the ability to differentiate individuals with ASD from those with non-ASD neuropsychiatric disorders with approximately

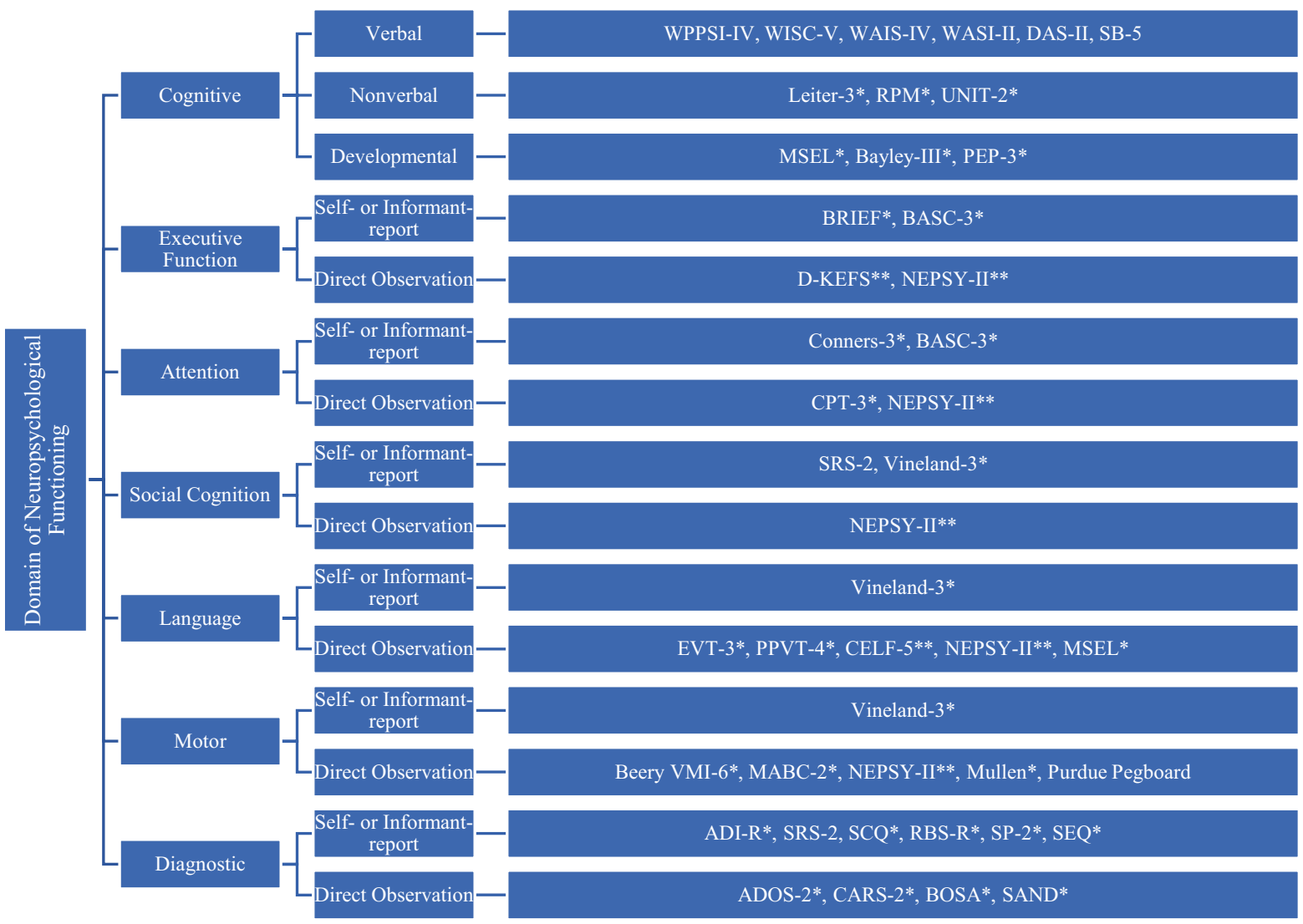

Note. Neuropsychological measures used to assess functioning in individuals with ASD.

* denotes measures appropriate for use with individuals with cognitive or language impairments; ** denotes subtests can be administered individually

Fig. 1 Developing neuropsychological testing batteries in ASD 
$96 \%$ specificity and $62 \%$ sensitivity [73]. These measures offer promising early data on advances in autism screening.

Specific measures can be used to capture restricted and repetitive behaviors (RRBs) in greater detail. The Repetitive Behavior Scale-Revised (RBS-R [74]), for example, is used to assess RRBs in individuals on the autism spectrum across six domains: Stereotyped Behavior, Self-injurious Behavior, Compulsive Behavior, Routine Behavior, Sameness Behavior, and Restricted Behavior. This self- and parent/caregiver-report questionnaire has also been normed on individuals with ID [74].

Sensory symptoms are also highly prevalent in individuals with ASD and are only briefly captured on the ADOS-2 and ADI-R. To aid in detailed evaluation of sensory symptoms several measures are available, including the Sensory Assessment for Neurodevelopmental Disorders (SAND [75]). The SAND is a clinician-administered direct observational assessment and corresponding caregiver interview that examines sensory hyperreactivity, hyporeactivity, and seeking behaviors across visual, tactile, and auditory modalities. The SAND is appropriate for individuals of all levels of ability. Several caregiver report questionnaires have also demonstrated utility in quantifying sensory symptoms in individuals with ASD. The Sensory Profile 2 (SP-2 [76]) and the Sensory Experiences Questionnaire (SEQ [77]) are widely used questionnaires normed for individuals with ASD that assess sensory processing patterns across different contexts. Overall, these measures meaningfully supplement diagnostic and neuropsychological information gathered during other aspects of the assessment process.

\section{Developing Neuropsychological Batteries}

Figure 1 provides a summary of neuropsychological assessment tools appropriate for individuals with ASD to help clinician's determine appropriate testing batteries for individuals with ASD of varying levels of functioning.

\section{Conclusions}

The field of clinical neuropsychology aims to examine the relationship between brain functioning and behavior in order to elucidate the nature and origin of an individual's strengths and weaknesses in domains such as cognition, attentional, executive, social-cognitive, language, and motor functioning. Given the heterogeneity of ASD and its diagnostic complexity, neuropsychological testing of individuals suspected or confirmed to have ASD requires clinical expertise to design personalized testing batteries and interpret results, while accounting for ASD symptomatology and common comorbid psychiatric presentations. Clarifying the relationship between ASD symptoms and other aspects of neuropsychological functioning is critical for differential diagnosis and for optimal treatment planning. We recommend thorough neuropsychological assessments should be used to aid in the differential diagnosis of common co-occurring conditions, such as ADHD, which requires standardized assessment for proper diagnosis. Neuropsychological evaluations are also an important method to monitor progress toward academic, occupational, and adaptive goals. Traditional clinician-administered neuropsychological assessments are often overlooked in the evaluation process of individuals with ASD and may be related to challenges such as access to expert neuropsychologists or psychologists with expertise in both ASD and neuropsychological assessment as well as costs associated with such evaluations. Specialized neuropsychological testing is costly and often not covered, or poorly covered, by insurance. Families in rural or isolated geographic areas may experience greater difficulty accessing qualified evaluators. In addition, individuals with ASD often fail to be referred for comprehensive neuropsychological evaluations due to a disconnection between the field of neuropsychology and the field of autism assessment in many clinical settings. Improving access to high-quality neuropsychological evaluations for individuals on the autism spectrum, of all levels of ability, is an important direction for the field.

\section{Compliance with ethical Standards}

Conflict of interest The authors declare no competing interests.

\section{References}

\section{Papers of particular interest, published recently, have been highlighted as:}

- Of importance

$\bullet$ Of major importance

1. American Psychiatric Association. Diagnostic and statistical manual of mental disorders. 5th ed. Washington, DC: American Psychiatric Association; 2013.

2. Maenner MJ, Shaw KA, Baio J, et al. Prevalence of autism spectrum disorder among children aged 8 years-Autism and Developmental Disabilities Monitoring Network, 11 Sites, United States, 2016. MMWR Surveill Summ. 2020;69(4):1-12.

3. Buck TR, Viskochil J, Farley M, Coon H, McMahon WM, Morgan J, Bilder DA. Psychiatric comorbidity and medication use in adults with autism spectrum. J Autism Dev Disord. 2014;44(12):3063-71. 
4. Lever AG, Geurts HM. Psychiatric co-occurring symptoms and disorders in young, middle-aged, and older adults with autism spectrum disorder. J Autism Dev Disord. 2016;46(6):1916-30.

5. Simonoff E, Pickles A, Charman T, Chandler S, Loucas T, Baird G. Psychiatric disorders in children with autism spectrum disorders: prevalence, comorbidity, and associated factors in a population-derived sample. J Am Acad Child Adolesc Psychiatry. 2008;47(8):921-9.

6. Lord C, Rutter M, DiLavore P, Risi S, Gotham K, \& Bishop $\mathrm{S}$, Autism Diagnostic Observation Schedule-2nd edition (ADOS-2). Western Psychological Corporation. 2012

7. Lord C, Rutter M, Le Couteur A. Autism Diagnostic InterviewRevised: a revised version of a diagnostic interview for caregivers of individuals with possible pervasive developmental disorders. J Autism Dev Disord. 1994;24(5):659-85.

8. Bishop SL, Farmer C, Thurm A. Measurement of nonverbal IQ in autism spectrum disorder: scores in young adulthood compared to early childhood. J Autism Dev Disord. 2015;45(4):966-74.

9. Wechsler D. Wechsler Adult Intelligence Scale-Fourth Edition (WAIS-IV). Pearson. 2008

10. Wechsler D. Wechsler Abbreviated Scale of Intelligence-Second Edition (WASI-II). Pearson. 2011

11. Wechsler D. Wechsler Preschool and Primary Scale of IntelligenceFourth Edition (WPPSI). Pearson. 2012

12. Wechsler, D. Wechsler Intelligence Scale for Children -Fifth Edition (WISC-V). Pearson. 2014

13. Zwick GP. Neuropsychological assessment in autism spectrum disorder and related conditions. Dialogues Clin Neurosci. 2017;19(4):373.

14. Lockyer L, \& Rutter M. A five- to fifteen-year follow-up study of infantile psychosis: IV. Patterns of cognitive ability. British Journal of Social and Clinical Psychology, 1970;9(2), 152-163.

15.• Takayanagi M, Kawasaki Y, Shinomiya M, Hiroshi H, Okada S, Ino T, \& Niwa SI. Review of cognitive characteristics of autism spectrum disorder using performance on six subtests on four versions of the Wechsler Intelligence Scale for Children. Journal of Autism and Developmental Disorders, 2021;1-14. A systematic review of research using the WISC found differing cognitive profiles in youth with ASD with and without co-occurring cognitive impairments.

16. Holdnack J, Goldstein G, Drozdick L. Social perception and WAISIV performance in adolescents and adults diagnosed with Asperger's syndrome and autism. Assessment. 2011;18(2):192-200.

17. Roid GH. Stanford-Binet Intelligence Scales-Fifth Edition. Riverside Publishing. 2003

18. Elliott CD. Differential Ability Scales-Second Edition (DAS-II). Harcourt Assessment. 2007

19. Baum KT, Shear PK, Howe SR, Bishop SL. A comparison of WISC-IV and SB-5 intelligence scores in adolescents with autism spectrum disorder. Autism. 2015;19(6):736-45.

20. Clements CC, Watkins MW, Schultz RT, Yerys BE. Does the factor structure of IQ differ between the Differential Ability Scales (DAS-II) normative sample and autistic children? Autism Res. 2020;13(7):1184-94.

21. Soorya L, Leon J, Trelles MP, Thurm A. Framework for assessing individuals with rare genetic disorders associated with profound intellectual and multiple disabilities (PIMD): the example of Phelan McDermid Syndrome. Clin Neuropsychol. 2018;32(7):1226-55.

22. Mullen, E. M. Mullen Scales of Early Learning. American Guidance Service Inc. 1995

23. Bayley N. Bayley Scales of Infant and Toddler DevelopmentThird Edition (Bayley-III). Harcourt Assessment. 2006

24. Schopler E, Lansing MD, Reichler RJ, Marcus LM. (2005) Psychoeducational Profile: TEACCH Individualized Assessment for Children with Autism Spectrum Disorders-Third Edition. Pro-Ed Inc.
25. Roid GH, \& Koch C. Leiter-3: Nonverbal cognitive and neuropsychological assessment. In Handbook of nonverbal assessment 2017; (pp. 127-150). Springer, Cham.

26. DiStefano C, Sadhwani A, Wheeler AC. Comprehensive assessment of individuals with significant levels of intellectual disability: challenges, strategies, and future directions. Am J Intellect Dev Disabil. 2020;125(6):434-48.

27. Farmer C, Golden C, \& Thurm A. Concurrent validity of the Differential Ability Scales, Second Edition, with the Mullen Scales of Early Learning in young children with and without neurodevelopmental disorders. Child Neuropsychology, 2016;22(5), 556-569.

28. Farmer C, Swineford L, Swedo SE, Thurm A. Classifying and characterizing the development of adaptive behavior in a naturalistic longitudinal study of young children with autism. J Neurodev Disord. 2018;10(1):1-9.

29. Kanne SM, Gerber AJ, Quirmbach LM, Sparrow SS, Cicchetti DV, Saulnier CA. The role of adaptive behavior in autism spectrum disorders: implications for functional outcome. J Autism Dev Disord. 2011;41(8):1007-18.

30. Klin A, Saulnier CA, Sparrow SS, Cicchetti DV, Volkmar FR, Lord C. Social and communication abilities and disabilities in higher functioning individuals with autism spectrum disorders: the Vineland and the ADOS. J Autism Dev Disord. 2007;37(4):748-59.

31. Sparrow SS, Cicchetti DV, Saulnier CA. Vineland Adaptive Behavior Scales, Third Edition (Vineland-3). Pearson. 2016

32. Farmer C, Adedipe D, Bal VH, Chlebowski C, Thurm A. Concordance of the Vineland Adaptive Behavior Scales, second and third editions. J Intellect Disabil Res. 2020;64(1):18-26.

33. Harrison PL, Oakland T. Adaptive Behavior Assessment System Manual-Third Edition. Psychological Corporation. 2015

34. Tamm L, Day HA, Duncan A. (2021) Comparison of adaptive functioning measures in adolescents with autism spectrum disorder without intellectual disability. Journal of Autism and Developmental Disorders, 1-10.

35. Lai CLE, Lau Z, Lui SS, Lok E, Tam V, Chan Q, Cheung EF. Meta-analysis of neuropsychological measures of executive functioning in children and adolescents with high-functioning autism spectrum disorder. Autism Res. 2017;10(5):911-39.

36. Demetriou EA, Lampit A, Quintana DS, Naismith SL, Song YJC, Pye JE, Guastella AJ. Autism spectrum disorders: a meta-analysis of executive function Mol Psychiatry 2018;23(5)1198-1204. A meta-analysis of executive function in ASD found significantly reduced executive function skills across six domains (Concept Formation/Set Shifting, Mental Flexibility/Set Switching, Fluency, Planning, Response Inhibition, and Working Memory) for individuals with ASD, as well as limited clinical sensitivity for measures of executive function in this population.

37. Delis DC, Kaplan E, Kramer JH. Delis Kaplan Executive Function System. Psychological Corporation. 2001a

38. Korkman M, Kirk U, Kemp S. NEPSY-II: A Developmental Neuropsychological Assessment. The Psychological Corporation. 2007

39. Latzman RD, Markon KE. The factor structure and age-related factorial invariance of the Delis-Kaplan Executive Function System (D-KEFS). Assessment. 2010;17(2):172-84.

40. McLean RL, Harrison AJ, Zimak E, Joseph RM, Morrow EM. Executive function in probands with autism with average IQ and their unaffected first-degree relatives. J Am Acad Child Adolesc Psychiatry. 2014;53(9):1001-9.

41. Gioia GA, Isquith PK, Guy SC, Kenworthy L. BRIEF: Behavior rating inventory of executive function. Psychological Assessment Resources. 2015

42. Antshel KM, Zhang-James Y, Wagner KE, Ledesma A, Faraone SV. An update on the comorbidity of ADHD and ASD: a focus on clinical management. Expert Rev Neurother. 2016;16(3):279-93. 
43. Ames CS, White SJ. Brief report: Are ADHD traits dissociable from the autistic profile? Links between cognition and behaviour. J Autism Dev Disord. 2011;41(3):357-63.

44. Ashwood KL, Tye C, Azadi B, Cartwright S, Asherson P, Bolton P. Brief report: adaptive functioning in children with ASD, ADHD and ASD+ ADHD. J Autism Dev Disord. 2015;45(7):2235-42.

45. $\mathrm{Ng}$ R, Heinrich K, Hodges EK. Brief report: Neuropsychological testing and informant-ratings of children with autism spectrum disorder, attention-deficit/ hyperactivity disorder, or comorbid diagnosis J Autism Dev Disord 2019;49(6)2589-2596. A study comparing neuropsychological outcomes in youth with ASD, ADHD, and ASD + ADHD that found group effects across clinician-administered and parent-report measures of attentional and social functioning.

46. Conners KC. Conners Rating Scales, 3rd Edition. Multi- Health Systems Inc. 2008

47. Conners KC. Conners' Continuous Performance Test II. MultiHealth Systems Inc. 2004

48. Constantino JN, Gruber CP. Social Responsiveness Scale: SRS-2. Western Psychological Services. 2012

49. Reynolds CR, Kamphaus RW. Behavior Assessment System for Children-Third Edition. NCS Pearson. 2015

50. Zhou X, Reynolds C, Zhu J, Kamphaus RW. Differentiating autism from ADHD in children and adolescents using BASC-3. Journal of Pediatric Neuropsychology. 2020;6:61-5.

51. Conners KC. Conners' Continuous Performance Test 3. MultiHealth Systems Inc. 2014

52. May T, Cornish K, Rinehart NJ. Gender profiles of behavioral attention in children with autism spectrum disorder. J Atten Disord. 2016;20(7):627-35.

53. Achenbach TM, Edelbrock C. Child Behavior Checklist. University of Vermont Department of Psychiatry. 1981

54. Bishop-Fitzpatrick L, Mazefsky CA, Eack SM, Minshew NJ. Correlates of social functioning in autism spectrum disorder: the role of social cognition. Research in Autism Spectrum Disorders. 2017;35:25-34.

55. Freeman LM, Locke J, Rotheram-Fuller E, Mandell D. Brief report: Examining executive and social functioning in elementary-aged children with autism. J Autism Dev Disord. 2017;47(6):1890-5.

56. Torske T, Nærland T, Øie MG, Stenberg N, Andreassen OA. Metacognitive aspects of executive function are highly associated with social functioning on parent-rated measures in children with autism spectrum disorder. Front Behav Neurosci. 2018;11:258.

57. Brooks BL, Sherman EM, Strauss E. NEPSY-II: a developmental neuropsychological assessment. Child Neuropsychol. 2009;16(1):80-101.

58. Hus V, Bishop S, Gotham K, Huerta M, Lord C. Factors influencing scores on the social responsiveness scale. J Child Psychol Psychiatry. 2013;54(2):216-24.

59. Tager-Flusberg H, Kasari C. Minimally verbal school-aged children with autism spectrum disorder: The neglected end of the spectrum. Autism Res. 2013;6(6):468-78.

60. Williams K. Expressive Vocabulary Test, $3^{\text {rd }}$ Edition (EVT-3). Pearson. 2018
61. Dunn DM. Peabody Picture Vocabulary Test, $5^{\text {th }}$ Edition (PPVT5). Pearson. 2018

62. Wiig EH, Secord WA, Semel E. Clinical Evaluation of Language Fundamentals: CELF-5. Pearson. 2013

63. Coret MC, McCrimmon AW, Test Review: Wiig EH, Semel E, Secord WA. Clinical Evaluation of Language Fundamentals-Fifth Edition (CELF-5). Journal of Psychoeducational Assessment, 2015;33(5), 495-500.

64. Ramos-Sánchez CP, Kortekaas D, Van Biesen D, Vancampfort D, Van Damme T. The relationship between motor skills and intelligence in children with autism spectrum disorder. Journal of Autism and Developmental Disorders, 2021;1-11.

65. Wilson RB, McCracken JT, Rinehart NJ, Jeste SS. What's missing in autism spectrum disorder motor assessments? J Neurodev Disord. 2018;10(1):1-13.

66. Beery KE, Beery NA, Buktenica NA. (2010) Beery-Buktenica Developmental Test of Visual-Motor Integration (6th ed.). NCS Pearson Inc.

67. Grace N, Johnson BP, Rinehart NJ, Enticott PG. Are motor control and regulation problems part of the ASD motor profile? A handwriting study Developmental Neuropsychology. 2018;43(7):581-94.

68. Green RR, Bigler ED, Froehlich A, Prigge MB, Travers BG, Cariello AN, Lainhart JE. Beery VMI performance in autism spectrum disorder. Child Neuropsychol. 2016;22(7):795-817.

69. Morrison S, Armitano CN, Raffaele CT, Deutsch SI, Neumann SA, Caracci H, Urbano MR. Neuromotor and cognitive responses of adults with autism spectrum disorder compared to neurotypical adults. Exp Brain Res. 2018;236(8):2321-32.

70. Schopler E, Van Bourgondien M, Wellman G, Love S. The Childhood Autism Rating Scale-Second Edition. Western Psychological Services. 2010

71. Rutter M, Bailey A, Lord C. The Social Communication Questionnaire. Western Psychological Services. 2003

72. Lord C, Holbrook A, Dow D, Byrne K, Grzadzinski R, Sterrett K, et al. Brief Observation of Symptoms Of Autism (BOSA) [unpublished manual]. Western Psychological Services. 2020

73. Sanchez MJ, Constantino JN. Expediting clinician assessment in the diagnosis of autism spectrum disorder. Dev Med Child Neurol. 2020;62(7):806-12.

74. Bodfish JW, Symons FJ, Parker DE, Lewis MH. Varieties of repetitive behavior in autism: Comparisons to mental retardation. J Autism Dev Disord. 2000;30(3):237-43.

75. Siper PM, Kolevzon A, Wang AT, Buxbaum JD, Tavassoli T. A clinician-administered observation and corresponding caregiver interview capturing DSM-5 sensory reactivity symptoms in children with ASD. Autism Res. 2017;10(6):1133-40.

76. Dunn W. Sensory Profile 2. Pearson. 2014

77. Baranek GT, David FJ, Poe MD, Stone WL, Watson LR. Sensory Experiences Questionnaire: discriminating sensory features in young children with autism, developmental delays, and typical development. J Child Psychol Psychiatry. 2006;47(6):591-601.

Publisher's Note Springer Nature remains neutral with regard to jurisdictional claims in published maps and institutional affiliations. 